\title{
GATA2 zinc finger 1 mutations are associated with distinct clinico-biological features and outcomes different from GATA2 zinc finger 2 mutations in adult acute myeloid leukemia
}

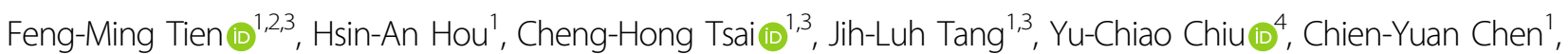
Yuan-Yeh Kuo ${ }^{5}$, Mei-Hsuan Tseng ${ }^{1}$, Yen-Ling Peng ${ }^{1}$, Ming-Chih Liư ${ }^{6}$, Chia-Wen Liu ${ }^{6}$, Xiu-Wen Liao ${ }^{3}$, Liang-In Lin? Chien-Ting Lin ${ }^{1,3}$, Shang-Ju Wu', Bor-Sheng Ko (1)', Szu-Chun Hsu', Shang-Yi Huang ${ }^{1}$, Ming Yao', Wen-Chien Chou ${ }^{1,8}$ and Hwei-Fang Tien ${ }^{1}$

\begin{abstract}
Mutations of the GATA binding protein 2 (GATA2) gene in myeloid malignancies usually cluster in the zinc finger 1 (ZF1) and the ZF2 domains. Mutations in different locations of GATA2 may have distinct impact on clinico-biological features and outcomes in AML patients, but little is known in this aspect. In this study, we explored GATA2 mutations in 693 de novo non-M3 AML patients and identified 44 GATA2 mutations in 43 (6.2\%) patients, including 31 in ZF1, 10 in ZF2, and three outside the two domains. Different from GATA2 ZF2 mutations, ZF1 mutations were closely associated

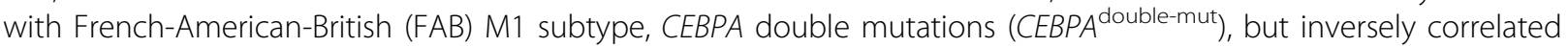
with FAB M4 subtype, NPM1 mutations, and FLT3-ITD. ZF1-mutated AML patients had a significantly longer overall survival (OS) than GATA2-wild patients and ZF2-mutated patients in total cohort as well as in those with intermediaterisk cytogenetics and normal karyotype. ZF1 mutations also predicted better disease-free survival and a trend of better OS in CEBPA double-mut patients. Sequential analysis showed GATA2 mutations could be acquired at relapse. In conclusion, GATA2 ZF1 mutations are associated with distinct clinico-biological features and predict better prognosis, different from ZF2 mutations, in AML patients.
\end{abstract}

\section{Introduction}

GATA binding protein 2 (GATA2) belongs to the GATA family of transcription factors which regulate hematopoietic stem cell proliferation and differentiation $^{1,2}$. GATA2 mutations have been reported in acute

\footnotetext{
Correspondence: H-A. Hou (hsinanhou@ntu.edu.tw) or

H-F. Tien (hftien@ntu.edu.tw)

'Division of Hematology, Department of Internal Medicine, National Taiwan University Hospital, Taipei, Taiwan

${ }^{2}$ Graduate Institute of Clinical Medicine, College of Medicine, National Taiwan University, Taipei, Taiwan

Full list of author information is available at the end of the article.
}

myeloid transformation of chronic myeloid leukemia $(\mathrm{CML})^{3}$, familial myelodysplastic syndrome-related acute myeloid leukemia (MDS/AML), pediatric $\mathrm{MDS}^{4,5}$, Emberger syndrome ${ }^{6}$, and monocytopenia and mycobacterial infection (MonoMAC) syndrome ${ }^{7,8}$. Mutations of GATA2 are also identified in AML patients, with an incidence varied from $3.6 \%$ in patients with FrenchAmerican-British (FAB) M5 subtype ${ }^{4}$ to $8.1-14.4 \%$ in non-selected AML patients ${ }^{9-11}$.

Somatic GATA2 mutations mainly cluster in the two zinc finger (ZF) domains, which can occupy GATA DNA motif 
in thousands of genes ${ }^{9}$. The patterns of somatic GATA2 mutations differ among myeloid diseases. ZF1 mutations predominate in AML, and ZF2 mutations are frequently identified in CML blastic phase ${ }^{3}$. GATA2 mutations are strongly associated with $C E B P A$ double mutations (CEB$\left.P A^{\text {double-mut }}\right)^{9,10,12}$. However, discrepancies exist among different reports regarding prognostic impact of GATA2 mutations in AML patients ${ }^{10,13}$. We hypothesize that mutations in different domains of GATA2 may have distinct impact on clinico-biological features and outcomes in AML patients, like $I D H 2$ mutations in which $I D H 2$ R172 is associated with gene mutations and clinical outcomes different from other IDH mutations ${ }^{14}$. However, little is known about this issue till now.

In this study, we investigated the clinical and prognostic relevance of mutations in different GATA2 domains in a large cohort of 693 unselected de novo non-M3 AML patients. To our knowledge, this is the first study to show GATA2 ZF1 mutations are associated with distinct clinical features, gene mutations, and outcomes different from ZF2 mutations. Longitudinal follow-ups were also performed in 419 samples from 124 patients to evaluate the dynamic changes of the mutations. Furthermore, we analyzed the global gene expression profiles in 328 patients to interrogate the possible molecular pathways associated with mutations in different GATA2 domains.

\section{Methods and materials}

\section{Subjects}

We consecutively enrolled 693 newly diagnosed de novo non-M3 AML patients at the National Taiwan University Hospital (NTUH) from 1994 to 2011. Diagnosis and classification of AML were made according to the FAB Cooperative Group Criteria and the 2016 WHO classification $^{15}$. To focus on a more homogeneous group of patients with de novo AML, those with antecedent hematological diseases, history of cytopenia, and family history of myeloid neoplasms or therapy-related AML were excluded ${ }^{16}$. Survival analyses were performed in 469 (67.7\%) patients who received standard chemotherapy. This study was approved by the Institutional Review Board of the NTUH, and written informed consents were obtained from all participants in accordance with the Declaration of Helsinki.

\section{Cytogenetics}

Chromosomal analyses were performed as described previously $^{17}$. Karyotypes were classified using Medical Research Council (MRC) risk groups ${ }^{18}$.

\section{Mutation analysis}

Mutation analysis of GATA2 exons $2-6^{12}$ and 20 other genes, including FLT3-ITD ${ }^{19}$, FLT3-TKD $^{19}, N R A S^{19}$,
$K R A S^{19}, K I T^{19}, P_{T P N 11^{20}}, C E B P A^{21}, R U N X 1^{22}, M L L-$ $\mathrm{PTD}^{23}$, ASXL1 $^{24}, I D H 1^{25}, I D H 2^{25}, T E T 2^{26}, D N M T 3 A^{16}$, $S F 3 B 1^{27}, S R S F 2^{27}, U 2 A F 1^{27}, N P M 1^{28}, W T 1^{29}, T P 53^{30}$, and $E T V 6^{31}$ were performed by Sanger sequencing as previously described for patients $(n=455)$ diagnosed from 1994 to 2007. For patients $(n=238)$ diagnosed after 2008, Ion torrent next-generation sequencing (NGS) (Thermo Fisher Scientific, MA, USA) was performed ${ }^{32}$. Serial analyses of mutations at diagnosis, complete remission (CR), and relapse were performed in 419 samples from 124 patients by targeted NGS using TruSight Myeloid Panel (Illumina, San Diego, CA, USA). HiSeq platform (Illumina) was used for sequencing with a median reading depth of $12,000 \times{ }^{32}$.

\section{Functional annotation analysis of GATA2 mutation- regulated genes}

We analyzed the differentially expression genes associated with GATA2 mutations by the knowledge-based Ingenuity Pathway Analysis (IPA) (Qiagen, Redwood City, CA) software for associated functions. We also used Gene Set Enrichment Analysis (GSEA) software to investigate systematic enrichments of GATA2 mutation-governed expressional profile in biological functions ${ }^{33}$. Statistical significance of the degree of enrichment was assessed by a 1000-time random permutation test.

\section{Statistical analysis}

The discrete variables were compared using the $\chi^{2}$ tests, but if the expected values of contingency tables were $<5$, Fisher's exact test was used. Mann-Whitney $U$ tests were used to compare continuous variables and medians of distributions. Overall survival (OS) was measured from the date of first diagnosis to the date of last follow-up or death from any cause. Disease-free survival (DFS) was measured from the date of diagnosis until treatment failure, relapse from $\mathrm{CR}$, or death from any cause, whichever occurred first. To ameliorate the influence of hematopoietic stem cell transplantation (HSCT) on survival, DFS and OS were censored at the time of HSCT in patients receiving the treatment ${ }^{34}$. Multivariate Cox proportional hazard regression analysis was used to investigate independent prognostic factors for OS and DFS. A $P$ value $<0.05$ was considered statistically significant. All statistical analyses were performed with the SPSS 18 (SPSS Inc., Chicago, IL, USA) and StatsDirect (Cheshire, England, UK).

\section{Results}

GATA2 mutations in patients with AML

Excluding two single-nucleotide polymorphisms $(\mathrm{A} 164 \mathrm{~T}, \mathrm{M} 400 \mathrm{~T})^{35}$ and eight missense mutations (N114T, M223I, P250A, A256V, L315P, C319F, V369A, S429T) 


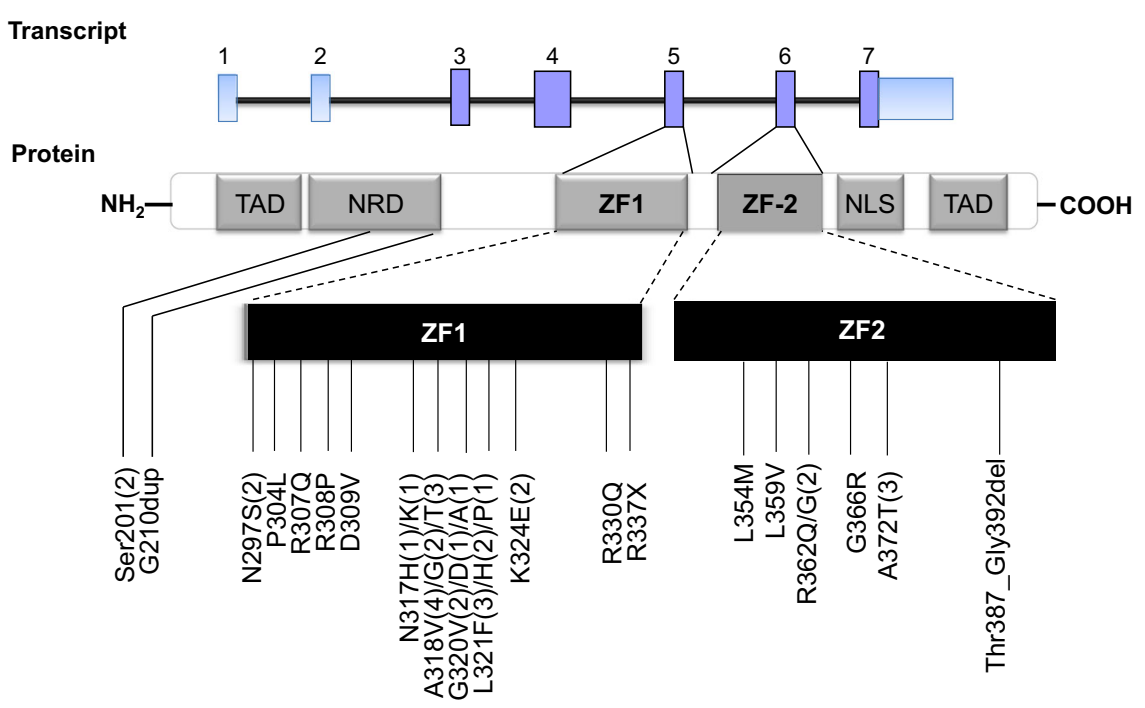

Fig. 1 Patterns and locations of the 44 GATA2 mutations

with unknown biologic significance (because they were not reported previously and could not be verified because of lack of matched bone marrow samples in CR), we identified 44 distinct GATA2 mutations in 43 (6.2\%) of 693 patients (Fig. 1). Forty GATA2 mutations were missense mutations. The other four were in-frame deletion or duplication: p.Ser201*(c.598_599insG) in two, p. Thr387_Gly392del (c.1160_1177delCCATGAAGAAGGA AGGGA) and G210dup (c.631_632insGCG) in one each. With regard to the functional sites, 31 mutations were clustered in the highly conserved $\mathrm{N}$-terminal ZF domain (ZF1 domain), and other 10 mutations were within Cterminal ZF domain (ZF2 domain). The remaining three mutations scattered outside of the ZF domains. The most common mutations were $\mathrm{A} 318 \mathrm{~V}(n=4)$, followed by L321F and A318T $(n=3$ each). p.Ser201* (c.598_599insG), N297S, A318G, G320V, L321H, and K324E occurred in two patients each. All other mutations were detected in only one patient each (Table 1). Only one patient had two GATA2 mutations (patient no. 20). All mutations were heterozygous. The mutant burden ranged from 4.89 to $52 \%$ with a median of $39.07 \%$ in ZF1 mutations, and from 10.74 to $50.26 \%$ with a median of $36.16 \%$ in ZF2 mutations.

\section{Correlation of GATA2 mutations with clinical and laboratory features}

Table 2 depicted the clinical characteristics of patients with and without GATA2 mutations. ZF1-mutated patients were younger (median, 39 years vs. 55 years, $P=0.004$ ), and had higher incidence of FAB M1 subtype (56.7\% vs. $22.1 \%, P<0.0001$ ), but lower incidence of FAB M4 subtype (3.3\% vs. $28.1 \%, P=0.003)$ than GATA2-wild patients. ZF1-mutated patients also had a higher incidence of FAB M1 subtype than ZF2-mutated patients $(P=0.044)$. The patients with ZF2 mutations showed similar clinical features to the GATA2-wild group, including peripheral white blood cell counts (median, 47.3 vs. $18.7 \mathrm{k} / \mu \mathrm{L})$, incidences of FAB M1 subtype (20\% vs. $22.1 \%$ ), and M4 subtype (20\% vs. $28.1 \%$ ).

\section{Association of GATA2 mutations with cytogenetics abnormalities}

Chromosome data were available in 669 patients at diagnosis, including 43 GATA2-mutated and 626 GATA2-wild patients (Supplementary Table 1). Totally, GATA2 mutations were closely associated with intermediate-risk cytogenetics. Compared to GATA2wild patients, ZF1-mutated patients had more intermediate-risk cytogenetics $(100 \%$ vs. $70.9 \%, P<$ $0.0001)$, normal karyotype ( $73.3 \%$ vs. $46.5 \%, P=0.004)$, and $\mathrm{t}(3 ; 3)(6.7 \%$ vs. $1.0 \%, P=0.048)$, but less favorablerisk $(0 \%$ vs. $13.6 \%, P=0.024)$ or unfavorable-risk cytogenetics ( $0 \%$ vs. $15.5 \%, P=0.014)$. There was no association of ZF1 mutations with other chromosomal abnormalities, including $+8,+11,+13$, and +21 .

\section{Association of GATA2 mutations with other molecular alterations}

To investigate the interaction of GATA2 ZF1 and ZF2 mutations with other genetic alterations in the pathogenesis of adult AML, a complete mutational screening of 20 other genes was performed. Only ZF1-mutated patients had a significantly higher frequency of $C E B P A^{\text {double-mut }}(66.7 \%$ vs. $6.7 \%, P<0.0001)$ than wildtype patients, but not ZF2-mutated patients (Table 3). ZF1-mutated patients had lower frequencies of NPM1 mutations ( $0 \%$ vs. $22 \%, P=0.004)$ and FLT3-ITD ( $4 \%$ vs. 
Table 1 The mutation patterns in 43 patients with GATA2 mutations at diagnosis

\begin{tabular}{|c|c|c|c|c|c|c|c|}
\hline \multirow[b]{2}{*}{ UPN } & \multirow[b]{2}{*}{ Age/sex } & \multirow[b]{2}{*}{ Karyotype } & \multirow[b]{2}{*}{ Location } & \multicolumn{3}{|l|}{ GATA2 mutations } & \multirow[b]{2}{*}{ Other mutations } \\
\hline & & & & DNA change & $\begin{array}{l}\text { Mutant } \\
\text { burden (\%) }\end{array}$ & Protein change & \\
\hline 1 & $29 \mathrm{~F}$ & $\mathrm{CN}$ & ZF1 & $c .953 C>T$ & 52 & A318V & $\begin{array}{l}\text { CEBPA }^{\mathrm{dm}}, \text { FLT3-ITD, } \\
\text { NRAS }\end{array}$ \\
\hline 2 & $40 \mathrm{M}$ & $\mathrm{CN}$ & ZF1 & $c .961 C>T$ & 49.37 & L321F & CEBPA $^{\mathrm{dm}}$, NRAS \\
\hline 3 & $65 F$ & $\begin{array}{l}\text { t(3;3)(q21;q26), del(12) } \\
(\text { p11p13) }\end{array}$ & ZF1 & $c .890 A>G$ & 49.04 & N297S & NRAS, ASXL1 \\
\hline 4 & $36 \mathrm{M}$ & $\mathrm{CN}$ & ZF1 & C.961C>T & 47.42 & L321F & CEBPA $^{\mathrm{dm}}$ \\
\hline 5 & $37 M$ & $-Y$ & ZF1 & c.959G $>\mathrm{T}$ & 47.19 & G320V & CEBPA $\mathrm{dm}^{\mathrm{m}}$ \\
\hline 6 & $36 \mathrm{M}$ & $\mathrm{CN}$ & ZF1 & c. $.970 A>G$ & 46.14 & K324E & CEBPA ${ }^{\mathrm{dm}}$, NRAS \\
\hline 7 & $27 M$ & $\mathrm{CN}$ & ZF1 & c. $953 C>G$ & 45.45 & A318G & CEBPA $^{\mathrm{dm}}$ \\
\hline 8 & $78 \mathrm{~F}$ & +8 & ZF1 & C. $1009 C>T$ & 45.3 & R337X & $\begin{array}{l}\text { FLT3-ITD, NRAS, } \\
\text { IDH2, SRSF2 }\end{array}$ \\
\hline 9 & $42 \mathrm{M}$ & $\begin{array}{l}\text { t(3;3)(q21;q26)/46, } \\
\text { idem,add(17)(p13) }\end{array}$ & ZF1 & c. $959 \mathrm{G}>\mathrm{A}$ & 44.62 & G320D & ASXL1, U2AF1 \\
\hline 10 & $34 \mathrm{M}$ & $\mathrm{CN}$ & $\mathrm{ZF1}$ & C. $962 T>A$ & 43.97 & $\mathrm{~L} 321 \mathrm{H}$ & $\begin{array}{l}\text { CEBPA }^{\text {sm }}, \text { NRAS, KIT, } \\
\text { IDH2, DNMT3A }\end{array}$ \\
\hline 11 & $20 \mathrm{~F}$ & $\mathrm{CN}$ & $\mathrm{ZF1}$ & c. $989 G>A$ & 43.14 & R330Q & $C^{\prime} E B P A^{\mathrm{dm}}, A S X L 1$ \\
\hline 12 & $32 \mathrm{M}$ & $\mathrm{CN}$ & ZF1 & c. $952 \mathrm{G}>\mathrm{A}$ & 42.99 & $\mathrm{~A} 318 \mathrm{~T}$ & CEBPA ${ }^{\mathrm{dm}}, \mathrm{KIT}$ \\
\hline 13 & $39 M$ & $\mathrm{CN}$ & ZF1 & $\mathrm{c} .911 \mathrm{C}>\mathrm{T}$ & 42.41 & P304L & MLL, TET2 \\
\hline 14 & $43 \mathrm{M}$ & $\mathrm{CN}$ & ZF1 & C. $.923 \mathrm{G}>\mathrm{C}$ & 41.21 & R308P & CEBPA $^{\mathrm{dm}}$, NRAS \\
\hline 15 & $18 \mathrm{M}$ & $\operatorname{del}(9)(q 22 q 34)$ & ZF1 & c.926A>G & 39.07 & D309V & $C E B P A^{\mathrm{sm}}$ \\
\hline 16 & $36 \mathrm{~F}$ & $\mathrm{CN}$ & ZF1 & c. $.920 G>A$ & 39.06 & R307Q & CEBPA ${ }^{\mathrm{dm}}$, NRAS \\
\hline 17 & $31 \mathrm{~F}$ & $\mathrm{CN}$ & ZF1 & $c .970 A>G$ & 37.98 & K324E & CEBPA $^{\mathrm{dm}}$ \\
\hline 18 & $55 \mathrm{M}$ & $\mathrm{CN}$ & ZF1 & c. $952 \mathrm{G}>\mathrm{A}$ & 32.72 & A318T & CEBPA $\mathrm{dm}^{\mathrm{m}}$ \\
\hline 19 & $69 F$ & $\mathrm{CN}$ & ZF1 & C.953C>T & 30.26 & A318V & CEBPA $A^{\mathrm{dm}}$ \\
\hline 20 & $57 M$ & $\mathrm{CN}$ & ZF1 & c.962T>A c.949A $>C$ & 23.94 & $\mathrm{~L} 321 \mathrm{H}, \mathrm{N} 317 \mathrm{H}$ & CEBPA ${ }^{\mathrm{dm}}$, TET2 \\
\hline 21 & $51 \mathrm{M}$ & +21 & $\mathrm{ZF1}$ & $c .953 C>T$ & 23.48 & A318V & CEBPA $^{\mathrm{dm}}, R \cup N X_{1}$ \\
\hline 22 & $39 F$ & $\begin{array}{l}46, X X, \operatorname{der}(3) t(3 ; 17) \\
(q 26 ; q 21), t(16 ; 17)(p 11 ; \\
q 11)\end{array}$ & ZF1 & C. $962 T>C$ & 20.48 & L321P & SF3B1 \\
\hline 23 & $82 \mathrm{M}$ & $\mathrm{CN}$ & ZF1 & c.951T>A & 20.46 & N317K & $R \cup N X 1, S F 3 B 1$ \\
\hline 24 & $19 F$ & $\mathrm{CN}$ & ZF1 & c.959G >C & 18.41 & G320A & CEBPA $^{\mathrm{dm}}$, FLT3-TKD \\
\hline 25 & $59 M$ & $\mathrm{CN}$ & ZF1 & C.953C >T & 18.15 & A318V & $C_{E B P A}{ }^{\mathrm{dm}}, N R A S$ \\
\hline 26 & $29 M$ & $\mathrm{CN}$ & ZF1 & $\mathrm{c.961C}>\mathrm{T}$ & 17.58 & L321F & $C E B P A^{\mathrm{sm}}$ \\
\hline 27 & $50 \mathrm{M}$ & $\mathrm{CN}$ & ZF1 & c.959G $>\mathrm{T}$ & 13.81 & G320V & $C_{E B P A^{\mathrm{dm}},}, U 2 A F 1$ \\
\hline 28 & $54 \mathrm{M}$ & $\mathrm{CN}$ & ZF1 & c. $953 C>G$ & 10.81 & A318G & CEBPA $^{\mathrm{dm}}$ \\
\hline 29 & $22 \mathrm{~F}$ & $\operatorname{del}(9 q)$ & ZF1 & c. $952 \mathrm{G}>\mathrm{A}$ & 6.02 & A318T & CEBPA $^{\mathrm{dm}}$ \\
\hline 30 & $78 \mathrm{M}$ & $\mathrm{CN}$ & ZF1 & $c .890 A>G$ & 4.89 & N297S & PTPN11, DNMT3A \\
\hline 31 & $76 \mathrm{M}$ & $\mathrm{CN}$ & $\mathrm{ZF2}$ & c. $1075 T>G$ & 50.26 & L359V & RUNX1 \\
\hline
\end{tabular}


Table 1 continued

\begin{tabular}{|c|c|c|c|c|c|c|c|}
\hline \multirow[b]{2}{*}{ UPN } & \multirow[b]{2}{*}{ Age/sex } & \multirow[b]{2}{*}{ Karyotype } & \multirow[b]{2}{*}{ Location } & \multicolumn{3}{|l|}{ GATA2 mutations } & \multirow[b]{2}{*}{ Other mutations } \\
\hline & & & & DNA change & $\begin{array}{l}\text { Mutant } \\
\text { burden (\%) }\end{array}$ & Protein change & \\
\hline 32 & $53 \mathrm{~F}$ & $\mathrm{CN}$ & $\mathrm{ZF2}$ & C. $1085 G>A$ & 48.68 & R362Q & $\begin{array}{l}\text { ASXL1, IDH2, } \\
\text { DNMT3A }\end{array}$ \\
\hline 33 & $28 \mathrm{~F}$ & $\mathrm{CN}$ & ZF2 & C. $1114 \mathrm{G}>\mathrm{A}$ & 46.82 & A372T & NPM1 \\
\hline 34 & $69 \mathrm{~F}$ & $\mathrm{CN}$ & ZF2 & c.1096G>A & 46.33 & G366R & NPM1 \\
\hline 35 & $18 \mathrm{~F}$ & $\mathrm{CN}$ & ZF2 & C. $1114 \mathrm{G}>\mathrm{A}$ & 39.8 & $\mathrm{~A} 372 \mathrm{~T}$ & NPM1, PTPN11 \\
\hline 36 & $20 \mathrm{M}$ & $\mathrm{CN}$ & ZF2 & C. $1084 C>G$ & 23.05 & R362G & $C E B P A^{s m}, A S X L 1$ \\
\hline 37 & $40 \mathrm{~F}$ & $\mathrm{t}(7 ; 11)$ & ZF2 & C. $1114 \mathrm{G}>\mathrm{A}$ & 21.36 & $\mathrm{~A} 372 \mathrm{~T}$ & FLT3-ITD, NRAS \\
\hline 38 & $60 M$ & $-Y$ & ZF2 & c. $1084 C>G$ & 32.52 & R362G & - \\
\hline 39 & $32 \mathrm{~F}$ & +10 & ZF2 & c.1160_1177delCCATGAAGAAGGAAGGGA & 17.59 & Thr387_Gly392del & CEBPA $^{\mathrm{dm}}$, NRAS \\
\hline 40 & $80 \mathrm{~F}$ & $\mathrm{CN}$ & $\mathrm{ZF} 2$ & C.1061C>T & 10.74 & T354M & CEBPA $A^{\mathrm{dm}}$, FLT3-ITD \\
\hline 41 & $71 \mathrm{M}$ & del(12)(p12p13), -7 & & C.598_599insG & 35.31 & Ser201 & $\begin{array}{l}\text { PTPN11, RUNX1, } \\
\text { ASXL1 }\end{array}$ \\
\hline 42 & $68 \mathrm{~F}$ & $\mathrm{CN}$ & & c.598_599insG & 34.1 & Ser201 & $\begin{array}{l}\text { FLT3-ITD, RUNXI, } \\
\text { MLL }\end{array}$ \\
\hline 43 & $76 \mathrm{M}$ & $\mathrm{CN}$ & & c.631_632insGCG & 40.36 & G210dup & TP53 \\
\hline
\end{tabular}

$U P N$ unique patient number, $C E B P A^{\mathrm{dm}}$ CEBPA double mutation, $C N$ cytogenetically normal, $Z F$ zinc finger

$19.9 \%, P=0.024)$ than wild-type patients. In contrast, ZF2-mutated patients had similar frequencies of NPM1 mutations (30\%) and FLT3-ITD (20\%) to those with wild type of GATA2. Both ZF1 and ZF2 mutations were mutually exclusive with KRAS, WT1, IDH1, TP53, and ETV6 mutations (Table 3).

\section{Impact of different GATA2 domains mutations on treatment response and clinical outcomes}

Of the 469 AML patients, including 27 GATA2 ZF1mutated and nine GATA2 ZF2-mutated patients, undergoing conventional intensive induction chemotherapy, 352 (75.1\%) patients achieved a CR. The CR rate was $85.2 \%$ in ZF1-mutated patients and $60 \%$ in ZF2-mutated patients (Table 2). The relapse rate was similar between the two groups.

With a median follow-up time of 78.6 months (ranges, 0.1-236 months), patients with GATA2 mutations as a whole had a trend of longer OS (5-year survival rate, $56 \%$ vs. $43 \%, P=0.078$ ) and DFS (median, 32.9 vs. 8.8 months, $P=$ 0.091 ) than those without GATA2 mutations (Supplementary Figure 1). Focusing on the prognostic implication of mutation sites, patients with GATA2 ZF1 mutations had a significantly better OS (5-year survival rate, $72 \%$ vs. $43 \%$, $P=0.003$ ) and DFS than GATA2-wild patients (median, 91.2 vs. 8.8 months, $P=0.022$ ) (Fig. 2). In contrast, patients with GATA2 ZF2 mutations had similar OS (5-year survival rate, $31 \%, P=0.297$ ) and DFS (median, 4.4 months, $P=$ 0.882 ) as the GATA2-wild group. Intriguingly, ZF1 mutations were also associated with better OS compared with ZF2 mutations $(P=0.001)$ (Fig. 2). In intermediate-risk cytogenetics group, ZF1-mutated patients had significantly superior OS (5-year survival rate, $72 \%$ vs. $39 \%, P=0.009$ ) and DFS (median, 91.2 vs. 7.8 months, $P=0.006$ ) than GATA2-wild patients, and a longer OS (5-year survival rate, $72 \%$ vs. $31 \%, P=0.007)$ and a trend toward longer DFS (median, 91.2 vs. 4.4 months, $P=0.133$ ) than ZF2-mutated patients (Fig. 3). The finding also held true in normal karyotype subgroup (Supplementary Figure 2). Multivariate analysis demonstrated that ZF1 mutation was an independent favorable prognostic factor for OS (HR 0.207, 95\% CI 0.066-0.652, $P=0.007$ ) and DFS (HR 0.529, 95\% CI 0.295-0.948, $P=0.032$ ) irrespective of age, white blood cell counts, cytogenetics, NPM1, and FLT3-ITD status. However, the prognostic independence of ZF1 mutation was lost if we included $C E B P A^{\text {double-mut }}$ as a covariable (Supplementary Table 2). We could not find the survival difference stratified by the degree of mutational burden in either ZF1 or ZF2-mutated patients (data not shown). Allo-HSCT in CR1 for ZF1-mutated patients did not offer survival benefit compared to postremission chemotherapy alone (data not shown). 
Table 2 Comparison of clinical and laboratory features between AML patients with GATA2 ZF1 domain and ZF2 domain mutations

\begin{tabular}{|c|c|c|c|c|c|c|c|}
\hline Variables & $\begin{array}{l}\text { GATA2-wild } \\
(n=650)\end{array}$ & $\begin{array}{l}\text { GATA2 mutations } \\
(n=43)\end{array}$ & $P$ value $^{\mathrm{a}}$ & $\begin{array}{l}\text { ZF1 domain mutations } \\
(n=30)\end{array}$ & $P$ value $^{\mathrm{b}}$ & $\begin{array}{l}\text { ZF2 domain mutations } \\
(n=10)\end{array}$ & $P$ value \\
\hline $\operatorname{Sex}^{\mathrm{d}}$ & & & 0.876 & & 0.291 & & 0.112 \\
\hline Male & $370(56.9)$ & $25(58.1)$ & & $20(66.7)$ & & $3(30)$ & \\
\hline Female & $280(43.1)$ & $18(41.9)$ & & $10(33.3)$ & & $7(70)$ & \\
\hline Age $\left(\right.$ year) ${ }^{\mathrm{e}}$ & $55(15-94)$ & $40(18-82)$ & 0.017 & $39(18-82)$ & 0.004 & $47(18-80)$ & 0.365 \\
\hline \multicolumn{8}{|l|}{$\mathrm{Lab}_{\text {data }}{ }^{\mathrm{e}}$} \\
\hline WBC $(k / \mu \mathrm{L})$ & $18.7(0.12-423)$ & $21.2(1.23-627.8)$ & 0.200 & $23.4(1.33-627.8)$ & 0.195 & $47.3(1.23-212.7)$ & 0.494 \\
\hline $\mathrm{Hb}(\mathrm{g} / \mathrm{dL})$ & $8.1(2.9-16.2)$ & $8.1(4.2-13.2)$ & 0.704 & $8.1(4.4-12.5)$ & 0.436 & $7.4(4.2-13.2)$ & 0.311 \\
\hline Platelet $(\mathrm{K} / \mu \mathrm{L})$ & $47(3-802)$ & $45(6-1017)$ & 0.565 & $47(6-1017)$ & 0.937 & $47(11-119)$ & 0.606 \\
\hline PB Blast(k/ $\mu \mathrm{L})$ & $7.33(0-371.9)$ & $9.09(0-456.7)$ & 0.077 & $11.3(0.06-456.7)$ & 0.067 & $29.9(0-140.7)$ & 0.358 \\
\hline $\mathrm{LDH}(\mathrm{U} / \mathrm{L})$ & 859 (206-15000) & 917 (299-4220) & 0.575 & $970(327-4220)$ & 0.385 & 1029 (394-2970) & 0.629 \\
\hline \multicolumn{8}{|l|}{$F A B^{d}$} \\
\hline Mo & $16(2.5)$ & $2(4.7)$ & 0.309 & $2(6.7)$ & 0.186 & $0(0)$ & $>0.999$ \\
\hline M1 & $144(22.1)$ & $21(48.8)$ & $<0.0001$ & $17(56.7)$ & $<0.0001$ & $2(20)$ & $>0.999$ \\
\hline M2 & 239 (36.8) & 17 (39.5) & 0.716 & $10(33.3)$ & 0.703 & $6(60)$ & 0.186 \\
\hline M4 & $183(28.1)$ & $3(7.0)$ & 0.002 & $1(3.3)$ & 0.003 & $2(20)$ & 0.734 \\
\hline M5 & $31(4.8)$ & $0(0)$ & 0.248 & $0(0)$ & 0.633 & $0(0)$ & $>0.999$ \\
\hline M6 & $27(4.2)$ & $0(0)$ & 0.403 & $0(0)$ & 0.625 & $0(0)$ & $>0.999$ \\
\hline Unclassified & $10(1.5)$ & $0(0)$ & $>0.999$ & $0(0)$ & $>0.999$ & $0(0)$ & $>0.999$ \\
\hline \multicolumn{8}{|c|}{2016 WHO classification ${ }^{d}$} \\
\hline $\mathrm{t}(8 ; 21)$ & $57(8.7)$ & $0(0)$ & 0.041 & $0(0)$ & 0.165 & $0(0)$ & $>0.999$ \\
\hline $\operatorname{lnv}(16)$ & $27(4.2)$ & $0(0)$ & 0.403 & $0(0)$ & 0.625 & $0(0)$ & $>0.999$ \\
\hline $\mathrm{t}(9 ; 11)$ & $9(1.4)$ & $0(0)$ & $>0.999$ & $0(0)$ & $>0.999$ & $0(0)$ & $>0.999$ \\
\hline$t(6 ; 9)$ & $3(0.5)$ & $0(0)$ & $>0.999$ & $0(0)$ & $>0.999$ & $0(0)$ & $>0.999$ \\
\hline $\operatorname{lnv}(3)$ & $1(0.2)$ & $2(4.6)$ & 0.011 & $2(6.7)$ & 0.005 & $0(0)$ & $>0.999$ \\
\hline $\mathrm{t}(1 ; 22)$ & $0(0)$ & $0(0)$ & - & $0(0)$ & - & $0(0)$ & - \\
\hline$C_{E B P A^{\mathrm{dm}}}$ & $43(6.6)$ & $22(51.2)$ & $<0.0001$ & $20(66.7)$ & $<0.0001$ & $2(20)$ & 0.144 \\
\hline NPM1 & $139(21.3)$ & $3(7.0)$ & 0.023 & $0(0)$ & 0.005 & $3(30)$ & 0.455 \\
\hline RUNX1 & $73(11.2)$ & $4(9.3)$ & $>0.999$ & $1(3.3)$ & 0.237 & $1(10)$ & $>0.999$ \\
\hline BCR-ABL & $1(0.2)$ & $0(0)$ & $>0.0999$ & $0(0)$ & $>0.999$ & $0(0)$ & $>0.999$ \\
\hline MRC & $93(14.3)$ & $0(0)$ & 0.008 & $0(0)$ & 0.025 & $0(0)$ & 0.372 \\
\hline AML, NOS & $204(31.4)$ & $12(27.9)$ & 0.633 & $7(23.3)$ & 0.351 & $4(40)$ & 0.516 \\
\hline Induction response ${ }^{f}$ & 431 & 38 & & 27 & & 9 & \\
\hline Complete remission & $323(74.9)$ & $29(76.3)$ & 0.851 & $23(85.2)$ & 0.230 & $5(60)$ & 0.241 \\
\hline Induction death & $32(7.4)$ & $1(2.6)$ & 0.503 & $0(0)$ & 0.243 & $1(10)$ & 0.508 \\
\hline Relapse & $161(49.8)$ & $9(31)$ & 0.052 & $8(34.8)$ & 0.163 & $1(16.7)$ & 0.371 \\
\hline
\end{tabular}

CEBPA ${ }^{\text {sm }}$ CEBPA single mutation, $C E B P A^{\mathrm{dm}}$ CEBPA double mutation, MRC myelodysplasia-related change, NOS not otherwise specified, $P B$ peripheral blood ${ }^{a} G A T A 2$-mutated patients vs. GATA2 wild-type patients

${ }^{\mathrm{b}}$ GATA2 ZF1-mutated patients vs. GATA2 wild-type patients

'GATA2 ZF2-mutated patients vs. GATA2 wild-type patients

${ }^{\mathrm{d}}$ Number of patients (\%)

'Median (range)

fOnly the 469 patients, including 27 with GATA2 ZF1 domain mutations, nine with GATA2 ZF2 domain mutations, and 431 without, who received conventional intensive induction chemotherapy and then consolidation chemotherapy if CR was achieved, as mentioned in the text, were included in the analysis

In $C E B P A^{\text {double-mut }}$ subgroup, GATA2 ZF1-mutated patients had a trend of longer OS (5-year survival rate, $76 \%$ vs. $68 \%, P=0.075)$ and a significantly longer DFS (median, 91.2 vs. 14.0 months, $P=0.034$ ) than GATA2wild patients (Fig. 4). ZF1 mutations allowed further refinement of the clinical outcome of $C E B P A^{\text {double-mut }}$ patients. The small number of ZF2-mutated patients $(n=3)$ in this group did not allow statistically meaningful correlations.

\section{Sequential studies of GATA2 mutations in AML patients}

GATA2 mutations were serially studied in 419 samples from 124 patients who had ever obtained a CR and had available samples for study, including 19 patients with and 105 patients without GATA2 mutations at diagnosis (Table 4). Among the 19 GATA2-mutated patients who had paired samples, all lost the original GATA2 mutations at remission. Five of the six patients regained the original GATA2 mutations at first relapse, but one (no. 27) lost the 
mutation. In the former five patients, the mutation burden, compared to that at diagnosis, was increased in one patient (no. 25), decreased in two (nos. 13 and 16), and stable in the remaining two (nos. 5 and 9). One patient (no. 9) retained the co-occurring ASXL1 mutations at CR status. Among the 105 patients who had no GATA2 mutations at diagnosis, four patients (nos. 44, 45, 46, and 47) acquired novel GATA2 mutations at relapse (Table 4).

\section{GATA2 expression and biological functions associated with} GATA2 mutations

We analyzed the microarray dataset of 328 patients studied to assess the impact of GATA2 mutations on gene expression and biological functions. By comparing the mRNA expression profiles between patients with and without GATA2 mutations, we found GATA2 expression levels were higher in those with GATA2 mutations $(P=$ 0.003). More specifically, both ZF1 and ZF2 mutations correlated with higher GATA2 expression level compared to GATA2 wild-type. GATA2 mutations were associated with significant differential expression of 159 probes ( $t$-test, $P<0.05$ and $>2$-fold change). IPA analysis revealed different molecular networks between the GATA2 ZF1 and ZF2-mutated group (Supplementary Figure 3). We also performed the GSEA analysis to identify biological functions associated with genes significantly enriched in GATA2-mutated AML, compared with GATA2-wild AML. Three-hundred and thirteen patients with wild-type GATA2, 12 patients with GATA2 ZF1 mutations, and three patients with GATA2 ZF2 mutations were analyzed. We identified significant underrepresentation of genes hyper-methylated in AML $(P=0.006 ;$ normalized enrichment score (NES) $=-1.49$; Supplementary Figure $4 \mathrm{~A})$ and genes related to apoptosis $(P=0.042$; NES $=$ -1.33 ) in the ZF1-mutated patients compared to GATA2 wild-type patients. ZF2-mutations were associated with

Table 3 Comparison of other genetic alterations between AML patients according to GATA2 mutation domain

\begin{tabular}{|c|c|c|c|c|c|c|c|c|c|}
\hline \multirow[t]{2}{*}{ Mutation } & \multirow[t]{2}{*}{ Total pts examined } & \multicolumn{5}{|c|}{ Pts with the other gene mutations (\%) } & \multirow[t]{2}{*}{$P$ value $^{\mathrm{a}}$} & \multirow[t]{2}{*}{$P$ value $^{b}$} & \multirow[t]{2}{*}{$P$ value $^{c}$} \\
\hline & & Whole cohort & GATA2 wt pts & GATA2 mutated pts & ZF1 & ZF2 & & & \\
\hline FLT3-ITD & 685 & 19.3 & 19.9 & 9.3 & 4.0 & 20 & 0.087 & 0.024 & $>0.999$ \\
\hline FLT3-TKD & 690 & 8.8 & 9.2 & 2.4 & 3.3 & 0 & 0.248 & 0.508 & $>0.999$ \\
\hline NRAS & 691 & 15.5 & 14.8 & 26.8 & 30 & 25 & 0.038 & 0.035 & 0.340 \\
\hline KRAS & 688 & 3.6 & 3.9 & 0 & 0 & 0 & 0.391 & 0.620 & $>0.999$ \\
\hline PTPN11 & 658 & 5 & 4.8 & 7.7 & 3.6 & 12.5 & 0.436 & $>0.999$ & 0.335 \\
\hline KIT & 690 & 4.8 & 4.8 & 4.9 & 6.7 & 0 & $>0.999$ & 0.652 & $>0.999$ \\
\hline WT1 & 688 & 6.8 & 7.3 & 0 & 0 & 0 & 0.103 & 0.257 & $>0.999$ \\
\hline NPM1 & 693 & 21.1 & 22 & 7 & 0 & 30 & 0.019 & 0.004 & 0.467 \\
\hline CEBPA & 689 & 14.2 & 11.1 & 60.5 & 76.7 & 30 & $<0.0001$ & $<0.0001$ & 0.095 \\
\hline$C E B P A^{d m}$ & 689 & 9.4 & 6.7 & 51.2 & 66.7 & 20 & $<0.0001$ & $<0.0001$ & 0.146 \\
\hline$R \cup N \times 1$ & 684 & 14 & 14.2 & 11.9 & 6.7 & 11.1 & 0.682 & 0.413 & $>0.999$ \\
\hline MLLPTD & 636 & 5.7 & 5.7 & 5.1 & 3.6 & 0 & $>0.999$ & $>0.999$ & $>0.999$ \\
\hline$A S X L 1$ & 691 & 14 & 14 & 14 & 10 & 20 & 0.987 & 0.786 & 0.640 \\
\hline $\mathrm{IDH} 1$ & 690 & 6.4 & 6.8 & 0 & 0 & 0 & 0.101 & 0.250 & $>0.999$ \\
\hline $\mathrm{IDH} 2$ & 691 & 12.7 & 13.1 & 7.1 & 6.7 & 11.1 & 0.262 & 0.410 & $>0.999$ \\
\hline TET2 & 670 & 11.9 & 12.4 & 4.9 & 6.9 & 0 & 0.212 & 0.562 & 0.610 \\
\hline DNMT3A & 685 & 17.4 & 18 & 7.3 & 6.9 & 11.1 & 0.080 & 0.124 & $>0.999$ \\
\hline TP53 & 685 & 7.7 & 8.1 & 2.4 & 0 & 0 & 0.241 & 0.158 & $>0.999$ \\
\hline ETV6 & 649 & 0.9 & 0.9 & 0 & 0 & 0 & $>0.999$ & $>0.999$ & $>0.999$ \\
\hline SF & 653 & 11.8 & 11.7 & 12.5 & 17.9 & 0 & 0.802 & 0.366 & 0.608 \\
\hline
\end{tabular}

Pts patients, CEBPA ${ }^{d m}$ CEBPA double-mutation, SF splicing factors, including SF3B1, SRSF2, and U2AF1

${ }^{a}$ GATA2-mutated patients vs. GATA2 wild-type patients

${ }^{\mathrm{b}}$ GATA2 ZF1-mutated patients vs. GATA2 wild-type patients

'GATA2 ZF2-mutated patients vs. GATA2 wild-type patients 
the Gene Oncology term of myeloid leukocyte differentiation $(P=0.03$; NES $=-1.46)$ (Supplementary Figure 4B). Comparing with ZF2-mutated AML, we identified significant overrepresentation of genes related to myeloid leukocyte differentiation $(P=0.042 ; \mathrm{NES}=1.36)$ and underrepresentation of genes hyper-methylated in AML $(P=0.029 ; \mathrm{NES}=-1.37)$ in the ZF1-mutated AML.

\section{Discussion}

To the best of our knowledge, this is the first study to explore differences in clinical and biological implications between the GATA2 ZF1 and ZF2 mutations in AML patients. We found that mutations in different domains were associated with distinct clinical features, co-occurring mutations and outcomes (Supplementary Table 3).
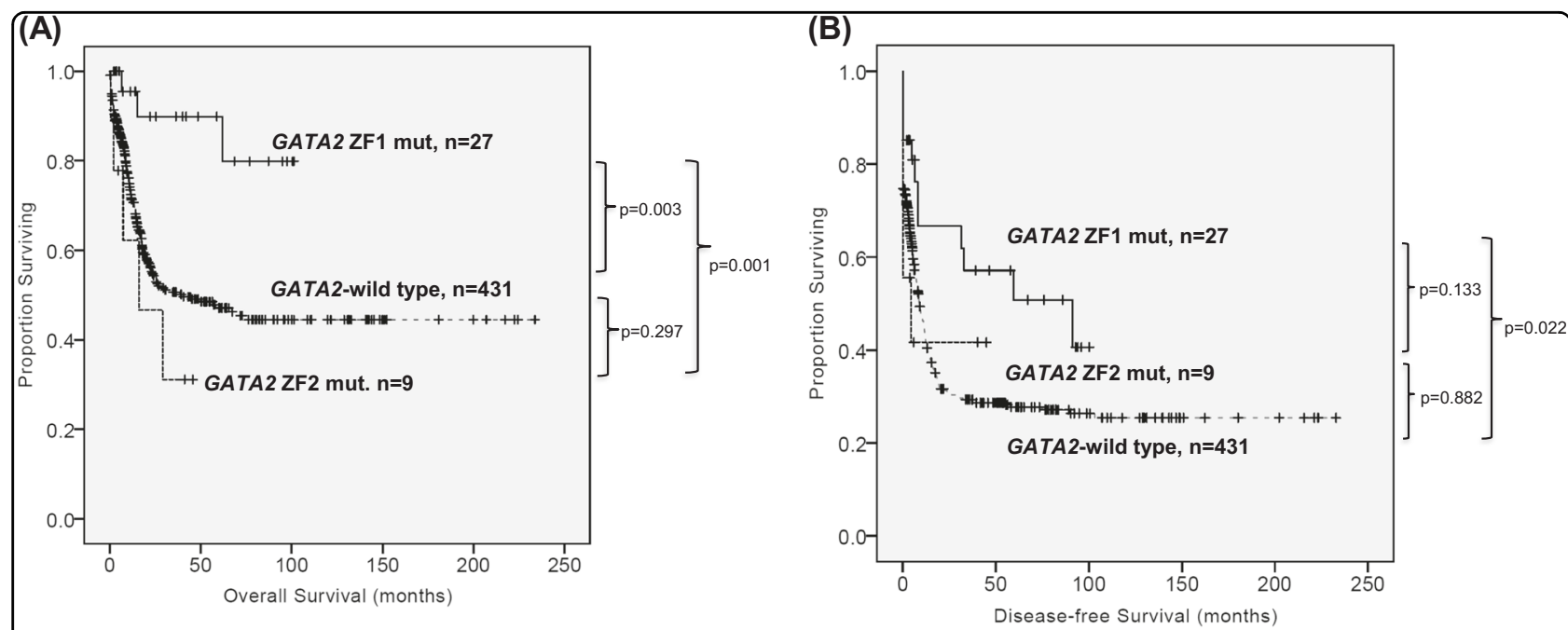

Fig. 2 Kaplan-Meier survival curves for OS (a) and DFS (b) stratified by the GATA2 mutation status and the sites of mutations in 467 AML patients who received standard intensive chemotherapy. Patients with GATA2 ZF1 mutations had a significantly better OS (5-year survival rate, $72 \%$ vs. $43 \%, P=0.003$ ) and DFS than GATA2-wild patients (median, 91.2 vs. 8.8 months, $P=0.022$ ). Patients with GATA2 ZF2 mutations had similar OS (5-year survival rate, $31 \%, P=0.297$ ) and DFS (median, 4.4 months, $P=0.882$ ) as the wild-type group. ZF1 mutations were also associated with better OS compared with ZF2 mutations $(P=0.001)$
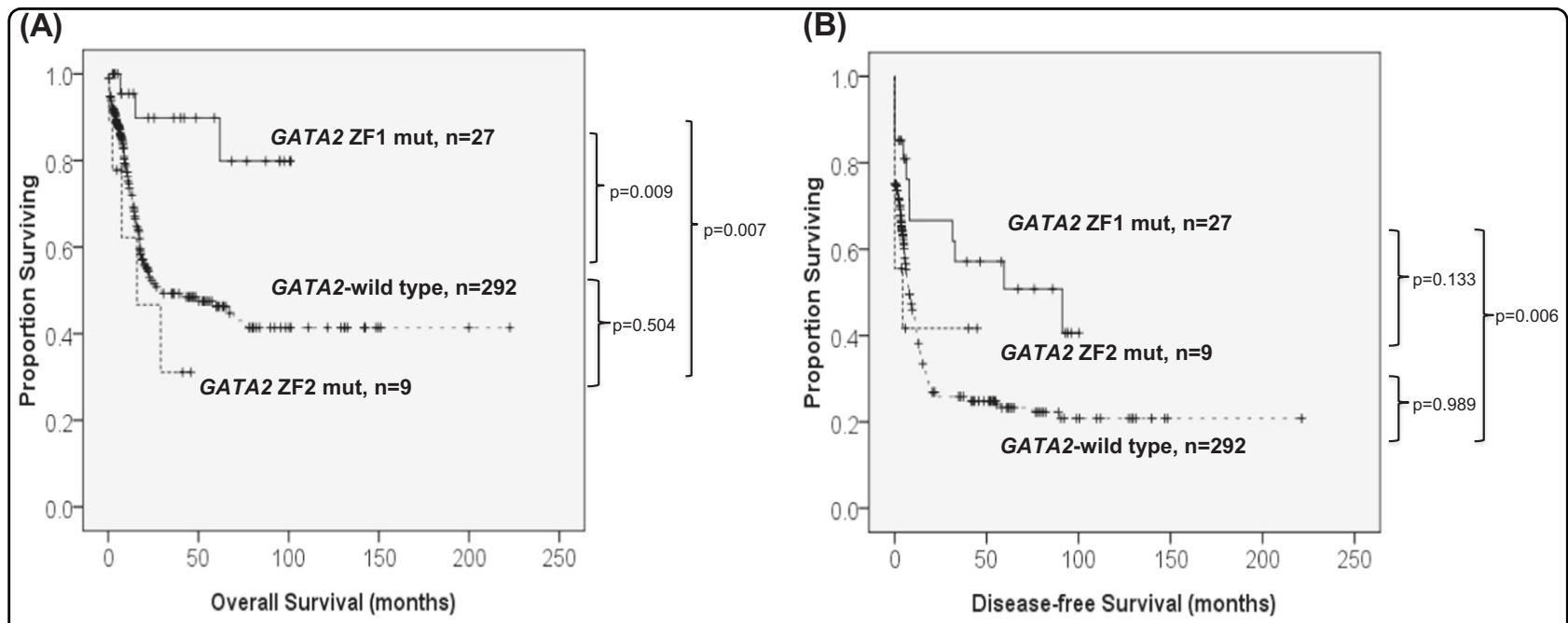

Fig. 3 Kaplan-Meier survival curves for OS (a) and DFS (b) stratified by the GATA2 mutation status and the sites of mutations in 328 intermediate-risk cytogenetics patients who received standard intensive chemotherapy. Patients with GATA2 ZF1 mutations had a significantly better OS (5-year survival rate, $72 \%$ vs. 39\%, $P=0.009$ ) and DFS (median, 91.2 vs. 7.8 months, $P=0.006$ ) than GATA2-wild patients. Patients with GATA2 ZF2 mutations had similar OS and DFS as the wild-type group ( $P=0.504, P=0.989$, respectively). ZF1 mutations were also associated with a longer OS (5-year survival rate, $72 \%$ vs. $31 \%, P=0.007$ ) and a trend toward longer DFS (median, 91.2 vs. 4.4 months, $P=0.133$ ) compared with ZF2 mutations 

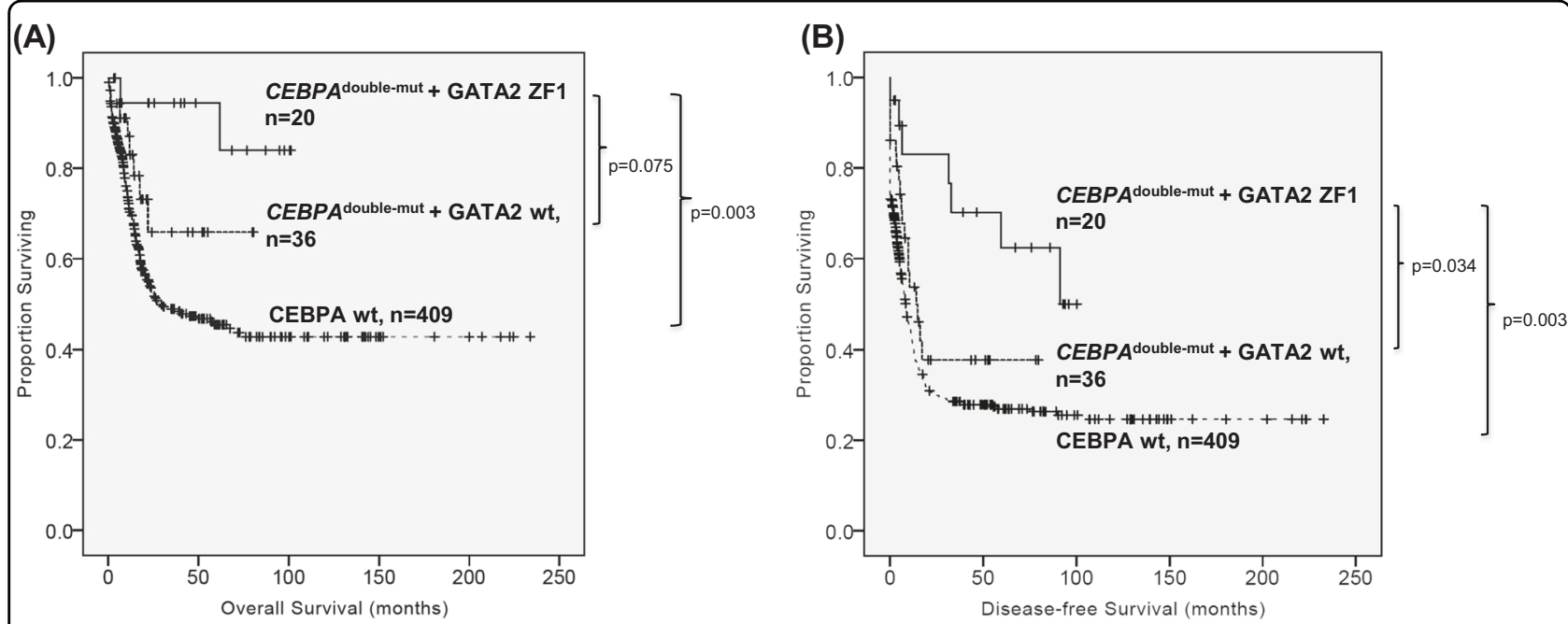

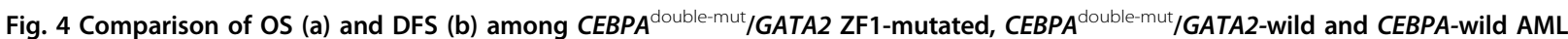
patients who received standard intensive chemotherapy. CEBPA ${ }^{\text {double-mut }}$ patients with GATA2 ZF1 mutations had a trend of longer OS (5-year survival rate, $76 \%$ vs. $68 \%, P=0.075$ ) and a significantly longer DFS (median, 91.2 vs. 14.0 months, $P=0.034$ ) that those with wild-type GATA2. The small number of ZF2-mutated patients in CEBPA ${ }^{\text {double-mut }}$ patients did not allow statistically meaningful correlations

The GATA2 mutation landscape in adult de novo AML differs from that in blastic crisis of $\mathrm{CML}^{3}$, familial MDS/ $\mathrm{AML}^{4}$, and pediatric $\mathrm{AML}^{5}$. In adult AML, ZF1 mutations predominate, while ZF2 mutations are reported sporadically ${ }^{10,36,37}$. In concordance with the findings, twothirds of the 44 distinct GATA2 mutations in our study were located in the ZF1 domain. We also reported two novel missense mutations in ZF2 domain (L359V and G366R) that had not been reported before in adult de novo AML patients, but ever identified in blastic crisis of CML.

AML with $C E B P A^{\text {double-mut }}$ has been included as a definite entity in the 2016 WHO Classification of Myeloid Neoplasms ${ }^{15}$. It is well established that GATA2 mutations frequently co-occur with $C E B P A^{\text {double-mut }}$ with an incidence of $18-41 \%^{9,10,12}$ and the two proteins show direct protein-protein interaction $^{38}$. Further study revealed GATA2 ZF1 mutants, but not the ZF2 L359V that is commonly seen at the progression of CML to blast crisis, had reduced capacity to enhance CEBPA-dependent activation of transcription ${ }^{9}$. Based on this functional study and the frequent co-occurrence of $C E B P A^{\text {double-mut }}$ and ZF1 mutations, but not ZF2 mutations, in AML patients, it is possible that GATA2 ZF1 mutations and $C E B P A^{\text {double-mut }}$ interact together to induce leukemogenesis. In addition, we found ZF1 mutations were associated with lower incidences of NPM1 mutations and FLT3-ITD than wild-type GATA2, different from ZF2 mutations as ZF2-mutated patients had similar incidences of these two mutations to those in GATA2-wild patients. GATA2 ZF1 and ZF2 mutations may induce AML through different oncogenic mechanisms and have distinct impact on clinical outcomes. Truly, in this study, we demonstrated that patients with GATA2 ZF1 mutations had a significantly longer OS than ZF2-mutated patients in total cohort, as well as in patients with intermediate-risk cytogenetics and normal karyotype.

The prognostic impact of GATA2 mutations in CEB$P A^{\text {double-mut }}$ patients was conflicting ${ }^{12,13,37,39}$. Greif et al. and Theis et al. found that GATA2 mutations did not impact clinical outcome in $C E B P A^{\text {double-mut }}$ patients. On the contrary, GATA2 mutations correlated with improved survival among $C E B P A^{\text {double-mut }}$ patients in other reports $^{12,13}$. In a study of Theis et al., 31 (74\%) of GATA2 mutations were detected in ZF1 domain, and 11 (26\%) in ZF2 domain. They did not show different clinical outcomes with respect to GATA2 ZF1 and ZF2 mutations in a cohort with both $C E B P A^{\text {double-mut }}$ and $C E B P A^{\text {single-mut }}$ patients $^{39}$. We were the first to investigate the prognostic implication of GATA2 ZF1 mutations in CEBPA double-mut patients and showed its association with a better DFS and a trend of longer OS than wild-type GATA2 among the $C E B P A^{\text {double-mut }}$ subgroup.

The poor prognostic impact of GATA2 ZF2 mutations was also witnessed in blast crisis CML patients as in de novo AML patients shown in this study ${ }^{4}$. The reason that ZF1 and ZF2 mutations had different survival impacts on de novo AML patients might be partially explained by their difference in association with $C E B P A^{\text {double-mut }}$, and by different oncogenic mechanisms. Further studies are warranted to explore the underlying mechanisms of the differences.

The study also recruited the largest number of de novo AML patients for sequential analyses of GATA2 
Table 4 Sequential studies in the AML patients with GATA2 mutations ${ }^{a}$

\begin{tabular}{|c|c|c|c|c|c|}
\hline UPN & Interval| (months) & Status & GATA2 mutations & Allele burden & Other mutations \\
\hline \multirow[t]{2}{*}{1} & & Initial & Ala318Val & 52 & CEBPA, FLT3-ITD, NRAS \\
\hline & 0.9 & CR1 & - & 0 & - \\
\hline \multirow[t]{2}{*}{4} & & Initial & Leu321Phe & 47.42 & CEBPA \\
\hline & 1.3 & CR1 & - & 0 & - \\
\hline \multirow[t]{4}{*}{5} & & Initial & Gly320Val & 47.19 & CEBPA \\
\hline & 6.6 & CR1 & - & 0 & - \\
\hline & 27.1 & Relapse1 & Gly320Val & 43.1 & CEBPA \\
\hline & 1.0 & CR2 & - & 0 & - \\
\hline \multirow[t]{2}{*}{6} & & Initial & Lys324Glu & 46.14 & CEBPA, NRAS \\
\hline & 0.9 & CR1 & - & 0 & - \\
\hline \multirow[t]{2}{*}{7} & & Initial & Ala318Gly & 45.45 & CEBPA \\
\hline & 0.9 & CR1 & - & 0 & - \\
\hline \multirow[t]{3}{*}{9} & & Initial & Gly320Asp & 44.62 & $A S X L 1$, U2AF1 \\
\hline & 3.2 & CR1 & - & 0 & ASXL1 \\
\hline & 6.5 & Relapse1 & Gly320Asp & 43.2 & ASXL1 1 \\
\hline \multirow[t]{2}{*}{12} & & Initial & Ala318Thr & 42.99 & CEBPA, KIT \\
\hline & 0.9 & CR1 & - & 0 & - \\
\hline \multirow[t]{3}{*}{13} & & Initial & Pro304Leu & 42.41 & MLL, TET2 \\
\hline & 3.5 & CR1 & - & 0 & - \\
\hline & 6.3 & Relapse1 & Pro304Leu & 3.2 & - \\
\hline \multirow[t]{2}{*}{14} & & Initial & Arg308Pro & 41.21 & CEBPA, NRAS \\
\hline & 1.4 & CR1 & - & 0 & - \\
\hline \multirow[t]{3}{*}{16} & & Initial & Arg307Gln & 39.06 & CEBPA, NRAS \\
\hline & 3.0 & CR1 & - & 0 & - \\
\hline & 34.7 & Relapse1 & $\operatorname{Arg} 307 \mathrm{Gln}$ & 27 & CEBPA \\
\hline \multirow[t]{2}{*}{18} & & Initial & Ala318Thr & 32.72 & CEBPA \\
\hline & 2.1 & CR1 & - & 0 & - \\
\hline \multirow[t]{3}{*}{20} & & Initial & Leu321His, Asn317His & 11.3 & CEBPA, TET2 \\
\hline & & & & 23.94 & \\
\hline & 1.4 & CR1 & - & 0,0 & - \\
\hline \multirow[t]{2}{*}{21} & & Initial & Ala318Val & 23.48 & CEBPA, RUNX1 \\
\hline & 1.0 & CR1 & - & 0 & - \\
\hline \multirow[t]{2}{*}{24} & & Initial & Gly320Ala & 18.41 & CEBPA, FLT3-TKD \\
\hline & 0.9 & CR1 & - & 0 & - \\
\hline \multirow[t]{3}{*}{25} & & Initial & Ala318Val & 18.15 & NRAS, CEBPA \\
\hline & 1.2 & CR1 & - & 0 & - \\
\hline & 12.0 & Relapse1 & Ala318Val & 43.5 & CEBPA \\
\hline \multirow[t]{2}{*}{27} & & Initial & Gly320Val & 13.81 & CEBPA, U2AF1 \\
\hline & 1.0 & CR1 & - & 0 & - \\
\hline
\end{tabular}


Table 4 continued

\begin{tabular}{|c|c|c|c|c|c|}
\hline UPN & Interval $^{\mathrm{b}}$ (months) & Status & GATA2 mutations & Allele burden & Other mutations \\
\hline & 3.5 & Relapse1 & - & 0 & CEBPA \\
\hline & 11.7 & CR2 & - & 0 & - \\
\hline & 5.9 & Relapse2 & - & 0 & CEBPA \\
\hline \multirow[t]{2}{*}{29} & & Initial & Ala318Thr & 6.02 & CEBPA \\
\hline & 1.0 & CR1 & - & 0 & \\
\hline \multirow[t]{2}{*}{39} & & Initial & Thr387_Gly392del & 17.59 & CEBPA, NRAS \\
\hline & 1.0 & CR1 & - & 0 & - \\
\hline \multirow[t]{2}{*}{41} & & Initial & Ser201 & 35.31 & PTPN11, RUNX1, ASXL1 \\
\hline & 0.8 & CR1 & - & 0 & - \\
\hline \multirow[t]{6}{*}{44} & & Initial & - & 0 & CEBPA, DNMT3A \\
\hline & 4.5 & CR1 & - & 0 & DNMT3A \\
\hline & 2.9 & Relapse1 & Glu180LysfsTer38 & 7.1 & DNMT3A \\
\hline & 1.1 & CR2 & - & 0 & - \\
\hline & 6.0 & Relapse2 & - & 0 & DNMT3A \\
\hline & 2.0 & CR3 & - & 0 & - \\
\hline \multirow[t]{5}{*}{45} & & Initial & - & 0 & DNMT3A, NPM1, NRAS, PTPN11 \\
\hline & 7.3 & CR1 & - & 0 & DNMT3A \\
\hline & 12.5 & Relapse1 & Arg307Leu & 5.6 & DNMT3A, NPM1 \\
\hline & 1.2 & CR2 & - & 0 & DNMT3A \\
\hline & 13.6 & Relapse2 & - & 0 & DNMT3A, NPM1 \\
\hline \multirow[t]{3}{*}{46} & & Initial & - & 0 & CEBPA \\
\hline & 2.9 & CR1 & - & 0 & - \\
\hline & 14.2 & Relapse1 & Leu321Pro & 26 & CEBPA \\
\hline \multirow[t]{7}{*}{47} & 29.0 & Initial & - & 0 & - \\
\hline & 1.0 & CR1 & - & 0 & - \\
\hline & 15.6 & Relapse1 & Gly320Asp & 15.9 & - \\
\hline & & & Leu321His & 15.1 & \\
\hline & 3.6 & CR2 & - & 0 & - \\
\hline & 11.8 & Relapse2 & Leu321His & 39.4 & - \\
\hline & 4.8 & CR3 & - & 0 & \\
\hline
\end{tabular}

UPN unique patient number, $C R$ complete remission, ND not done, "-" negative

${ }^{a}$ The results of serial studies in 101 patients without GATA2 mutation at both diagnosis and relapse were not shown in this table

${ }^{b}$ Interval between the two successive statuses

mutations by NGS during clinical follow-ups. The original mutations in all 19 GATA2-mutated patients were lost at remission status, confirming them to be truly somatic mutations. We showed GATA2 mutation was not stable during disease evolution. One (no. 27) of the six patients with GATA2 mutations at diagnosis lost the mutation at relapse. Among the 105 patients who had no GATA2 mutations at diagnosis, four (nos. 44, 45, 46, 47) acquired novel GATA2 mutations at relapse. The four mutations were all ZF1 mutations.

In conclusion, GATA2 ZF1 mutations, but not ZF2 mutations, are closely associated with $C E B P A^{\text {double-mut }}$, and inversely correlated with NPM1 mutations and FLT3ITD. The two GATA2 ZF domain mutations have different impacts on OS in AML patients. GATA2 ZF1 mutations also affect clinical outcome in $C E B P A^{\text {double-mut }}$ 
patients. Incorporation of GATA2 ZF1, not ZF2 mutations, allows further refinement of the WHO Classification in the specific entity of AML with $C E B P A^{\text {double-mut }}$.

\section{Acknowledgements}

We would like to acknowledge the service provided by the DNA Sequencing Core of the First Core Laboratory, National Taiwan University College of Medicine. This work was partially sponsored by grants MOST 104-2314-B-002128-MY4 and 106-2314-B-002-226-MY3 from the Ministry of Science and Technology (Taiwan), and MOHW 107-TDU-B-211-114009 from the Ministry of Health and Welfare (Taiwan), NTUH 102P06, from the Department of Medical Research, National Taiwan University Hospital, and Taiwan Health Foundation.

\section{Authors' contributions}

F.-M.T. was responsible for literature collection, data management and interpretation, statistical analysis, and manuscript writing; H.-A.H. was responsible for study design, plan and coordination, literature collection, data management and interpretation, statistical analysis, and manuscript writing; C.-H. T. and Y.-C.C. were responsible for bioinformatics analysis; L.I.L. and Y.-Y.K. were responsible for mutation analysis and interpretation; C.-T.L., C.-Y.C., W.-C. C., M.-Y., S.-Y.H., J.-L.T., B.-S.K., S.-C.H., S.-J.W., X.-W.L., and W.T. contributed patient samples and clinical data; Y.-L.P., M.-H.T., C.-W.L., and M.-C.L. performed the gene mutation and chromosomal studies; and H.F.T. planned, designed, coordinated the study over the entire period, and wrote the manuscript.

\section{Author details}

'Division of Hematology, Department of Internal Medicine, National Taiwan University Hospital, Taipei, Taiwan. ${ }^{2}$ Graduate Institute of Clinical Medicine, College of Medicine, National Taiwan University, Taipei, Taiwan. ${ }^{3}$ Tai-Cheng Stem Cell Therapy Center, National Taiwan University, Taipei, Taiwan. ${ }^{4}$ Greehey Children's Cancer Research Institute, University of Texas Health Science Center at San Antonio, San Antonio, TX, USA. ${ }^{5}$ Graduate Institute of Oncology, College of Medicine, National Taiwan University, Taipei, Taiwan. ${ }^{6}$ Department of Pathology, National Taiwan University Hospital, Taipei, Taiwan. ${ }^{7}$ Department of Clinical Laboratory Sciences and Medical Biotechnology, College of Medicine, National Taiwan University, Taipei, Taiwan. ${ }^{8}$ Department of Laboratory Medicine, National Taiwan University Hospital, Taipei, Taiwan

\section{Conflict of interest}

The authors declare that they have no conflict of interest.

\section{Publisher's note}

Springer Nature remains neutral with regard to jurisdictional claims in published maps and institutional affiliations.

Supplementary Information accompanies this paper at (https://doi.org/ 10.1038/s41408-018-0123-2).

Received: 4 June 2018 Revised: 20 July 2018 Accepted: 1 August 2018 Published online: 31 August 2018

\section{References}

1. Tsai, F. Y. et al. An early haematopoietic defect in mice lacking the transcription factor GATA-2. Nature 371, 221-226 (1994).

2. Tsai, F. Y. \& Orkin, S. H. Transcription factor GATA-2 is required for proliferation/ survival of early hematopoietic cells and mast cell formation, but not for erythroid and myeloid terminal differentiation. Blood 89, 3636-3643 (1997).

3. Zhang, S. J. et al. Gain-of-function mutation of GATA-2 in acute myeloid transformation of chronic myeloid leukemia. Proc. Natl Acad. Sci. USA 105, 2076-2081 (2008)

4. Hahn, C. N. et al. Heritable GATA2 mutations associated with familial myelodysplastic syndrome and acute myeloid leukemia. Nat. Genet. 43, 1012-1017 (2011).
5. Wlodarski, M. W. et al. Prevalence, clinical characteristics, and prognosis of GATA2-related myelodysplastic syndromes in children and adolescents. Blood 127, 1387-1397 (2016).

6. Ostergaard, P. et al. Mutations in GATA2 cause primary lymphedema associated with a predisposition to acute myeloid leukemia (Emberger syndrome). Nat. Genet. 43, 929-931 (2011).

7. Hsu, A. P. et al. Mutations in GATA2 are associated with the autosomal dominant and sporadic monocytopenia and mycobacterial infection (MonoMAC) syndrome. Blood 118, 2653-2655 (2011).

8. Kazenwadel, J. et al. Loss-of-function germline GATA2 mutations in patients with MDS/AML or MonoMAC syndrome and primary lymphedema reveal a key role for GATA2 in the lymphatic vasculature. Blood 119 1283-1291 (2012).

9. Greif, P. A. et al. GATA2 zinc finger 1 mutations associated with biallelic CEBPA mutations define a unique genetic entity of acute myeloid leukemia. Blood 120, 395-403 (2012).

10. Fasan, A. et al. GATA2 mutations are frequent in intermediate-risk karyotype AML with biallelic CEBPA mutations and are associated with favorable prognosis. Leukemia 27, 482-485 (2013).

11. Green, C. L. et al. GATA2 mutations in sporadic and familial acute myeloid leukaemia patients with CEBPA mutations. Br. J. Haematol. 161, 701-705 (2013).

12. Hou, H. A. et al. GATA2 mutations in patients with acute myeloid leukemiapaired samples analyses show that the mutation is unstable during disease evolution. Ann. Hematol. 94, 211-221 (2015).

13. Grossmann, $V$. et al. CEBPA double-mutated acute myeloid leukaemia harbours concomitant molecular mutations in $76.8 \%$ of cases with TET2 and GATA2 alterations impacting prognosis. Br. J. Haematol. 161, 649-658 (2013).

14. Papaemmanuil, E. et al. Genomic classification and prognosis in acute myeloid leukemia. N. Engl. J. Med. 374, 2209-2221 (2016).

15. Arber, D. A. et al. The2016 revision to the World Health Organization classification of myeloid neoplasms and acute leukemia. Blood 127, 2391-2405 (2016).

16. Hou, H. A. et al. DNMT3A mutations in acute myeloid leukemia: stability during disease evolution and clinical implications. Blood 119, 559-568 (2012).

17. Hou, H. A. et al. Incorporation of mutations in five genes in the revised International Prognostic Scoring System can improve risk stratification in the patients with myelodysplastic syndrome. Blood Cancer J. 8, 39 (2018).

18. Grimwade, D. et al. The importance of diagnostic cytogenetics on outcome in AML: analysis of 1,612 patients entered into the MRC AML 10 trial. The Medical Research Council Adult and Children's Leukaemia Working Parties. Blood 92 2322-2333 (1998)

19. Hou, H. A. et al. Clinical implications of the SETBP1 mutation in patients with primary myelodysplastic syndrome and its stability during disease progression. Am. J. Hematol. 89, 181-186 (2014).

20. Hou, H. A. et al. Characterization of acute myeloid leukemia with PTPN11 mutation: the mutation is closely associated with NPM1 mutation but inversely related to FLT3/ITD. Leukemia 22, 1075-1078 (2008).

21. Lin, L. I. et al. Characterization of CEBPA mutations in acute myeloid leukemia: most patients with CEBPA mutations have biallelic mutations and show a distinct immunophenotype of the leukemic cells. Clin. Cancer Res. 11, 1372-1379 (2005)

22. Tang, J. L. et al. AML1/RUNX1 mutations in 470 adult patients with de novo acute myeloid leukemia: prognostic implication and interaction with other gene alterations. Blood 114, 5352-5361 (2009).

23. Shiah, H. S. et al. Clinical and biological implications of partial tandem duplication of the MLL gene in acute myeloid leukemia without chromosomal abnormalities at 11q23. Leukemia 16, 196-202 (2002).

24. Chen, T. C. et al. Dynamics of ASXL1 mutation and other associated genetic alterations during disease progression in patients with primary myelodysplastic syndrome. Blood Cancer J. 4, e177 (2014).

25. Lin, C. C. et al. IDH mutations are closely associated with mutations of DNMT3A, ASXL1 and SRSF2 in patients with myelodysplastic syndromes and are stable during disease evolution. Am. J. Hematol. 89, 137-144 (2014).

26. Chou, W. C et al. TET2 mutation is an unfavorable prognostic factor in acute myeloid leukemia patients with intermediate-risk cytogenetics. Blood $\mathbf{1 1 8}$, 3803-3810 (2011)

27. Hou, H. A. et al. Splicing factor mutations predict poor prognosis in patients with de novo acute myeloid leukemia. Oncotarget 7, 9084-9101 (2016). 
28. Falini, B. et al. Cytoplasmic nucleophosmin in acute myelogenous leukemia with a normal karyotype. N. Engl. J. Med. 352, 254-266 (2005).

29. Hou, H. A. et al. WT1 mutation in 470 adult patients with acute myeloid leukemia: stability during disease evolution and implication of its incorporation into a survival scoring system. Blood 115, 5222-5231 (2010).

30. Hou, H. A. et al. TP53 mutations in de novo acute myeloid leukemia patients: longitudinal follow-ups show the mutation is stable during disease evolution. Blood Cancer J. 5, e331 (2015).

31. Silva, F. P. et al. ETV6 mutations and loss in AML-MO. Leukemia 22, 1639-1643 (2008).

32. Tsai, C. H. et al. Prognostic impacts and dynamic changes of cohesin complex gene mutations in de novo acute myeloid leukemia. Blood Cancer J. 7, 663 (2017).

33. Subramanian, A. et al. Gene set enrichment analysis: a knowledge-based approach for interpreting genome-wide expression profiles. Proc. Natl Acad. Sci. USA 102, 15545-15550 (2005).
34. Hou, H. A. et al. Integration of cytogenetic and molecular alterations in risk stratification of 318 patients with de novo non-M3 acute myeloid leukemia Leukemia 28, 50-58 (2014).

35. Richards, S. et al. Standards and quidelines for the interpretation of sequence variants: a joint consensus recommendation of the American College of Medical Genetics and Genomics and the Association for Molecular Pathology. Genet. Med. 17, 405-424 (2015).

36. Niimi, K. et al. GATA2 zinc finger 2 mutation found in acute myeloid leukemia impairs myeloid differentiation. Leuk. Res. Rep. 2, 21-25 (2013).

37. Marceau-Renaut, A. et al. Classification of CEBPA mutated acute myeloid leukemia by GATA2 mutations. Am. J. Hematol. 90, E93-E94 (2015).

38. Tong, Q., Tsai, J., Tan, G., Dalgin, G. \& Hotamisligil, G. S. Interaction between GATA and the C/EBP family of transcription factors is critical in GATA-mediated suppression of adipocyte differentiation. Mol. Cell. Biol. 25, 706-715 (2005).

39. Theis, F. et al. Clinical impact of GATA2 mutations in acute myeloid leukemia patients harboring CEBPA mutations: a study of the AML study group. Leukemia 30, 2248-2250 (2016). 